\title{
Inhibition of COX-2 Impairs Colon Cancer Liver Metastasis through Reduced Stromal Cell Reaction
}

\author{
Alba Herrero, Aitor Benedicto*, Irene Romayor, Elvira Olaso and Beatriz Arteta \\ Department of Cell Biology and Histology, University of the Basque Country, School of Medicine and Nursing, Leioa 48940, Bizkaia, \\ Spain
}

\begin{abstract}
Liver colonization is initiated through the interplay between tumor cells and adhesion molecules present in liver sinusoidal endothelial cells (LSECs). This crosstalk stimulates tumor COX-2 upregulation and $\mathrm{PGE}_{2}$ secretion. To elucidate the role of the LSEC intercellular adhesion molecule-1 (ICAM-1) in the prometastatic response exerted by tumor and stromal COX-2, we utilized celecoxib (CLX) as a COX-2 inhibitory agent. We analyzed the in vitro proliferative and secretory responses of murine C26 colorectal cancer (CRC) cells to soluble ICAM-1 (sICAM-1), cultured alone or with LSECs, and their effect on LSEC and hepatic stellate cell (HSC) migration and in vivo liver metastasis. CLX reduced sICAM-1-stimulated COX-2 activation and PGE 2 secretion in C26 cells cultured alone or cocultured with LSECs. Moreover, CLX abrogated sICAM-1-induced C26 cell proliferation and C26 secretion of promigratory factors for LSECs and HSCs. Interestingly, CLX reduced the protumoral response of HSC, reducing their migratory potential when stimulated with C26 secretomes and impairing their secretion of chemotactic factors for LSECs and C26 cells and proliferative factors for C26 cells. In vivo, CLX abrogated the prometastatic ability of sICAM-1-activated C26 cells while reducing liver metastasis. COX-2 inhibition blocked the creation of a favorable tumor microenvironment (TME) by hindering the intratumoral recruitment of activated HSCs and macrophages in addition to the accumulation of fibrillar collagen. These results point to COX-2 being a key modulator of processes initiated by host ICAM-1 during tumor cell/LSEC/HSC crosstalk, leading to the creation of a prometastatic TME in the liver.
\end{abstract}

Key Words: Liver metastasis, Tumor microenvironment, Cyclooxygenase-2, Hepatic stellate cells, CAF, Colorectal cancer

\section{INTRODUCTION}

Colorectal cancer (CRC) is currently one of the most prevalent malignant diseases (Siegel et al., 2019). While the primary tumor is commonly resected, dissemination of the primary malignancy to distant organs worsens prognosis, with the liver being the most common target organ for CRC cells (van der Pool et al., 2012). Such a scenario further complicates treatment strategies and, thus, negatively impacts life expectancy. Therefore, understanding the organ-specific prometastatic events that occur in tumor colonization of the liver arises as one of the most urgent requirements for coping with this disease.

CRC cell invasion of the liver starts at the hepatic sinusoids-narrow, sinuose, and highly specialized capillaries composed of hepatic-specific non-parenchymal cell types, such as liver sinusoidal endothelial cells (LSECs), resident macrophages (also known as Kupffer cells (KC)), and pericyte-like hepatic stellate cells (HSCs). The interaction of CRC cells with LSECs via adhesion molecules is one of the earliest events that initiate the prometastatic cascade. As a result, a proinflammatory and proangiogenic microenvironment is generated (Khatib et al., 2002; Arteta et al., 2010).

Intercellular adhesion molecule-1 (ICAM-1) is one of the adhesion molecules proposed as a key factor triggering this initial response. ICAM-1 is constitutively expressed in LSECs and is upregulated under inflammatory conditions (Volpes et al., 1990; Benedicto et al., 2019). We have previously shown a reduction in the metastatic burden of the liver when preventing tumor lymphocyte function associated antigen-1 (LFA-1) for interacting with its ligand, ICAM-1, on LSECs in an experimental metastatic model of CRC cells (Benedicto et al., 2017, 2019).

In the early phases of experimental liver colonization of CRC

\section{Open Access https://doi.org/10.4062/biomolther.2020.160}

This is an Open Access article distributed under the terms of the Creative Commons Attribution Non-Commercial License (http://creativecommons.org/licenses/by-nc/4.0/) which permits unrestricted non-commercial use, distribution, and reproduction in any medium, provided the original work is properly cited.
Received Sep 13, 2020 Revised Dec 3, 2020 Accepted Dec 9, 2020

Published Online Jan 18, 2021

\section{${ }^{*}$ Corresponding Author}

E-mail: aitor.benedicto@ehu.es

Tel: +34-94-601-3301, Fax: +34-94-601-3266 
cells, ICAM-1 prompts the secretion of different inflammatory mediators, such as IL-1 $\beta, I L-6, \mathrm{PGE}_{2}$, and TNF- $\alpha$ (Benedicto et al., 2019). Interestingly, cyclooxigenase-2 (COX-2), the limiting enzyme in the synthesis of $\mathrm{PGE}_{2}$, is one of the intracellular mediators of several inflammatory molecules (Greenhough et al., 2009) and it has been related to CRC and disease progression. In this regard, Shoji et al. (2003) showed that the implantation of CRC xenografts increased COX-2 expression in cancer cells, while elevated COX-2 expression in human malignant tissue was related to lymph node and liver metastasis (Yu et al., 2005). Several studies showed that COX-2 inhibition reduces the metastatic spread of CRC cells (Chen et al., 2001; Nagatsuka et al., 2002; Yao et al., 2004). In recent years, COX-2 inhibitors have been postulated as good candidates for the treatment of liver metastasis in combination with antiangiogenic drugs (Xu et al., 2014). Increasing evidence indicates that recruitment of host cells to developing metastatic foci are key in the secretion of proangiogenic vascular endothelial growth factor (VEGF) through their interaction with cancer cells and, therefore, the promotion of tumor growth (Olaso et al., 2003; Benedicto et al., 2019).

Despite this plethora of information, little is known about the role of COX-2 as a major force in the creation of the proinflammatory and proangiogenic microenvironment that boosts tumor progression during liver metastasis by means of resident cell recruitment. In this study, we aimed to shed some light on the ICAM-1-dependent COX-2-driven mechanisms during cancer cell/LSEC crosstalk in the early stage of liver colonization. Furthermore, we analyzed, for the first time, the COX2 dependence of the prometastatic response of HSCs in the hepatic microenvironment generated by colonizing $\mathrm{CRC}$ cells.

\section{MATERIALS AND METHODS}

\section{Animals}

BALB/c mice, 6-8 weeks old, were obtained from Charles River (Barcelona, Spain). Maintenance, care, and experimental conditions were developed under institutional guidelines and national laws for experimental animal care. All of the proceedings were approved by the Basque Country University Ethical Committee (CEID) in accordance with institutional, national, and international guidelines regarding the protection and care of animals used for scientific purposes.

\section{Cell lines}

In vivo assays were conducted using murine C26 colon adenocarcinoma cell line, syngenic with BALB/c, that was obtained from ATCC (LGC Standards S.L.U., Barcelona, Spain). In addition, for the in vitro experiments, mouse origin $\mathrm{NIH}$ 3T3 fibroblast and J774A.1 macrophage cell lines were used, both obtained from ATCC. All of the cells lines were cultured under standard conditions, C26 in RPMI-1640, NIH 3T3 in DMEM/F-12, and J774A.1 in DMEM, all of which were supplemented with $10 \%$ heat-inactivated fetal bovine serum (FBS), penicillin $(100 \mathrm{U} / \mathrm{mL})$, streptomycin $(100 \mu \mathrm{L})$, and amphotericin B $(25 \mu \mathrm{g} / \mathrm{mL}$; Thermo Fisher Scientific, Waltham, MA, USA).

\section{C26 secretomes}

Tumor cell secretomes were obtained by culturing $5.3 \times 10^{4}$ C26 tumor cells $/ \mathrm{cm}^{2}$ on 24-wells plates in complete RPMI1640 medium overnight. Then, the media was replaced with fresh serum-free RPMI-1640 medium. After $24 \mathrm{~h}$ of incubation, the media was collected and centrifuged at $4000 \mathrm{rpm}$ for 5 min for the collection of $\mathrm{C} 26$ secretome. For COX-2 inhibition, cells were treated for $1 \mathrm{~h}$ with $20 \mu \mathrm{M}$ celecoxib (CLX; SigmaAldrich, St. Louis, MO, USA) in 1\% FBS containing RPMI1640. Afterwards, the cells were activated as indicated with sICAM-1 (200 ng/mL; Life Technologies, Carlsbad, CA, USA) in $1 \%$ FBS containing RPMI-1640 for $18 \mathrm{~h}$. Finally, the medium was replaced with serum-free RPMI-1640 medium and collected after $24 \mathrm{~h}$, followed by $4000 \mathrm{rpm}$ centrifugation for 5 $\mathrm{min}$, and the supernatant was stored at $-20^{\circ} \mathrm{C}$.

\section{Isolation and culture of primary LSECs and HSCs}

The isolation and culture of mouse LSECs and HSCs have been described elsewhere (Smedsrod and Pertoft, 1985). Briefly, livers were perfused using collagenase type IV, disaggregated, and were subjected to isopycnic centrifugation using a Percoll gradient (GE Healthcare, Chicago, IL, USA). $\mathrm{KCs}$ were removed by differential adhesion onto plastic. The obtained LSECs were cultured onto 24-well plates coated with $0.05 \mathrm{mg} / \mathrm{mL}$ collagen type I (Thermo Fisher Scientific) at $1.6 \times 10^{6} \mathrm{cell} / \mathrm{mL}$ in RPMI-1640 supplemented with $5 \%$ FBS. LSECs were incubated for at least $2 \mathrm{~h}$ at $37^{\circ} \mathrm{C}$ prior to the start of experiments. HSCs were cultured in RPMI-1640 in uncoated plates for $18 \mathrm{~h}$ before experimentation. For the COX-2 inhibition assays, HSCs were treated with $20 \mu \mathrm{M} \mathrm{CLX}$ for $1 \mathrm{~h}$ before adding any treatment routine. C26 were pretreated with $20 \mu \mathrm{M}$ CLX before sICAM-1 activation before their addition to LSEC cultures.

\section{Establishment of LSEC cocultures with C26 tumor cells}

After isolation, LSECs were incubated for $3 \mathrm{~h}$ with RPMI1640 supplemented with 5\% FBS before experimentation. Next, the $\mathrm{C} 26$ cells were added to a 1:5 LSEC/tumor cell ratio with RPMI-1640 supplemented with 1\% FBS for $6 \mathrm{~h}$. In some experiments, the tumor cells were subjected to either ICAM-1 stimulation, COX-2 inhibition, or both before tumor cell addition to LSEC cultures.

\section{ELISA assays}

The presence of $\mathrm{PGE}_{2}$ and VEGF were analyzed through an enzyme-linked immunosorbent assay (ELISA) in cell culture supernatants following the manufacturer's instructions (R\&D Systems, Minneapolis, MN, USA).

\section{COX-2 activity measure}

For the quantification of COX-2 activity, either cell monocultures or cocultures treated under the different experimental conditions were incubated for $30 \mathrm{~min}$ in the presence of arachidonic acid $(10 \mu \mathrm{M})$, and the culture supernatant was collected for the analysis of $\mathrm{PGE}_{2}$ through ELISA.

\section{Proliferation assay}

For measurement of tumor cell proliferation, $1.56 \times 10^{4}$ tumor cells $/ \mathrm{cm}^{2}$ were cultured for $24 \mathrm{~h}$. Cells were treated with $20 \mu \mathrm{M} \mathrm{CLX}$ for $1 \mathrm{~h}$. The media was changed to fresh media containing $1 \%$ FBS and supplemented with increasing concentrations of sICAM-1, ranging from 50 up to $200 \mathrm{ng} / \mathrm{mL}$. Cell proliferation was measured after 24 and $48 \mathrm{~h}$ using an MTT assay (Sigma-Aldrich). 


\section{Transwell migration assay}

For the transwell migration assay, $2 \times 10^{4}$ cells/transwell of either LSECs or HSCs were cultured for $3 \mathrm{~h}$ in type I collagencoated modified Boyden chambers (Greiner BioOne, Kremsmünster, Austria). Afterward, the cells were stimulated with different secretome preparations for $18 \mathrm{~h}$ before analysis of the cell migration. The inserts were cut off, fixed in 4\% PFA, and stained with crystal violet (Sigma-Aldrich). For the quantification, 5-10 images were obtained at 200× magnification from each insert and were counted using ImageJ software $(\mathrm{NIH}$, Bethesda, MD, USA).

\section{Wound healing assay}

Fibroblasts and macrophages were cultured overnight at $2 \times 10^{5}$ cells $/ \mathrm{mL}$ on 24 -well plates in DMEM-F12 and DMEM medium, respectively, supplemented with $10 \%$ FBS. The next day, cells were treated with mitomycin C (Fisher Scientific, Thermo Fisher Scientific; $5 \mu \mathrm{g} / \mathrm{mL}$ for fibroblasts and $1 \mu \mathrm{g} / \mathrm{mL}$ for macrophages) for $2 \mathrm{~h}$. Afterwards, a scratch or wound was created using a $200 \mu \mathrm{L}$ tip, and the medium was replaced with C26 secretome obtained after different treatments, diluted at a ratio of $1: 2$ in fresh RPMI, and supplemented with $1 \%$ FBS. Pictures were taken at 0 and $24 \mathrm{~h}$. Quantification was carried out using ImageJ software $(\mathrm{NIH})$ through the measurement of total wound area after $24 \mathrm{~h}$ compared with that at $0 \mathrm{~h}$.

\section{Experimental development of hepatic metastasis}

A total of $2 \times 10^{5}$ tumor cells diluted in PBS were injected intrasplenically (i.s.) in BALB/c mice anesthetized with Nembutal $(50 \mathrm{mg} / \mathrm{kg}$ ). The mice were randomly separated into four groups as follows: control tumor-bearing mice group, control tumor-bearing CLX-treated mice group, sICAM-activated tumor-bearing mice group, and SICAM-activated tumor-bearing CLX-treated mice group. CLX was administered daily through oral cleavage for $14 \mathrm{~d}(100 \mu \mathrm{g} / \mathrm{kg})$. Then, the livers were collected and fixed in a zinc solution and embedded in paraffin for histological analysis. The metastatic liver area occupied by the tumor was quantified through H\&E staining in $5 \mu \mathrm{m}$ thick liver sections. Furthermore, the collagen deposition was analyzed through Picrosirius Red staining. All in vivo experiments were carried out twice with a minimum of 5 mice per experimental group.

\section{Immunohistochemical assays}

Recruitment of the host cells was analyzed in the liver tissue by immunohistochemical analysis through alpha-smooth muscle actin (ASMA) and F4/80 labeling for HSCs and macrophages, respectively. For ASMA staining, $5 \mu \mathrm{m}$ liver tissue sections were incubated at $95^{\circ} \mathrm{C}$ for $30 \mathrm{~min}$ in a citrate buffer ( $\mathrm{pH}$ 6.0) for antigen retrieval. Next, endogenous peroxidase was blocked through 40 min of incubation in $3 \% \mathrm{H}_{2} \mathrm{O}_{2}$ containing $1 \times$ PBS, followed by $1 \mathrm{~h}$ of incubation in $5 \%$ FBS containing $1 \times$ PBS. Then, the samples were incubated with $F(a b)$ antibody to inhibit unspecific binding, followed by overnight incubation with the mouse anti-human ASMA antibody (1:500; DakoCytomation, Agilent Technologies, Santa Clara, CA, USA). Next, the samples were washed with $1 \times$ PBS, incubated with rat anti-mouse secondary antibody (1:500; Invitrogen, Thermo Fisher Scientific), and visualized through HRP (1:500) and DAB Quanto substrate (Thermo Fisher Scientific). For the F4/80 staining, antigen retrieval was carried out by incubating the samples with $100 \mu \mathrm{L}$ of proteinase $\mathrm{K}$ (final concentration
$1.2 \mathrm{U} / \mathrm{mL}$; Sigma-Aldrich) diluted in $1 \mathrm{~mL}$ of TE buffer $(0.5 \%$ Triton X-100 diluted in $50 \mathrm{mM}$ Tris with $1 \mathrm{mM}$ EDTA). After peroxidase and unspecific binding blocking, as described above, the liver tissue sections were incubated overnight with rat antimouse F4/80 primary antibody (1:200; Bio-Rad, Hercules, CA, USA). After incubation with a biotin-conjugated secondary goat-anti rat antibody (1:500; Invitrogen, Thermo Fisher Scientific), the process proceeded as described above.

\section{Statistical analyses}

Data are expressed as mean \pm standard deviation (SD) of three independent experiments. The statistical analysis was performed using SPSS version 13.0 (Professional statistic, Chicago, IL, USA). Individual comparisons were performed using a two-tailed, unpaired Student's $t$-test.

\section{RESULTS}

\section{COX-2 inhibition impairs tumor cell proliferation mediated by ICAM-1}

The enhancement of uncontrolled tumor growth provided by the host microenvironment is a hallmark of metastasis. We have previously shown that $\mathrm{C} 26$ cell colonization of the liver correlates with ICAM-1 upregulation on LSECs (Arteta et al., 2010; Benedicto et al., 2019). The interplay between metastatic C26 cells and LSECs in the liver sinusoids was mimicked by stimulating C 26 cells with sICAM- 1 for up to 48 h. sICAM-1 heightened $\mathrm{C} 26$ cell proliferation by nearly two-fold after 24 $\mathrm{h}$, and was maintained for the following $24 \mathrm{~h}$. Interestingly, COX-2 inhibition by CLX uncovered that this early tumor response to sICAM-1 is partly dependent on tumor COX-2 (Fig. 1). COX-2 inhibition exerted a discrete effect on the basal proliferation of C26 cells after $24 \mathrm{~h}$, while completely abrogated sICAM-1-stimulated C26 cell proliferation. However, after 48 $\mathrm{h}$, both the basal and sICAM-1-induced proliferation of C26 cells was inhibited.

\section{CRC cell interaction with LSECs drives tumor COX-2 synthesis of $\mathrm{PGE}_{2}$ through endothelial ICAM-1}

Early steps of liver colonization occur in the liver sinusoids, and the interaction of tumor cells with LSECs represent a limiting step for the development of liver metastasis. Our group has previously shown that this interaction is highly dependent on ICAM-1 (Arteta et al., 2010; Benedicto et al., 2019). We revealed that $\mathrm{C} 26$ activation by SICAM-1 induced a stimulation similar to that observed after direct interaction with LSECs (Arteta et al., 2010). Here, we show that tumor cells increase the secretion of the proinflammatory mediator $\mathrm{PGE}_{2}$ upon sICAM-1 activation in a COX-2-dependent manner (Fig. 2A, 2B). In line with this approach, coculture experiments of tumor cells and freshly isolated primary LSECs revealed similar results, with a two-fold $\mathrm{PGE}_{2}$ enhancement in the C26-LSEC coculture secretomes (Fig. 2C). The $\mathrm{PGE}_{2}$ increase was paralleled by a substantial two-fold upregulation in COX-2 activity (Fig. 2D). Interestingly, CLX downregulated both COX-2 activity and $\mathrm{PGE}_{2}$ secretion in sICAM-1-activated $\mathrm{C} 26$ cells cultured alone or in coculture with LSECs. Therefore, tumor cell COX-2 and $\mathrm{PGE}_{2}$ production may represent a key mediator during the interplay between C26 cancer cells and LSECs, representing the starting point for early liver colonization of CRC. 

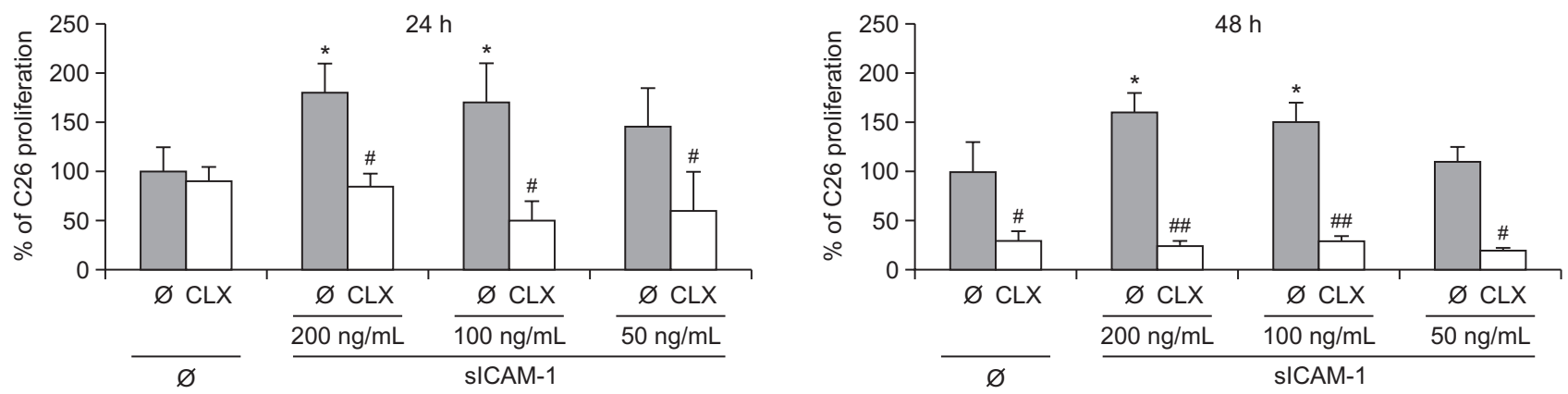

Fig. 1. Effect of COX-2 inhibition on tumor cell proliferation. The effect of COX-2 inhibition was analyzed in untreated colorectal cancer (CRC) cells and upon stimulation with recombinant sICAM-1 for 24 and $48 \mathrm{~h}$, untreated or treated with $20 \mu \mathrm{M}$ celecoxib (CLX). Tumor cells were treated with CLX for $1 \mathrm{~h}$ and stimulated with sICAM-1 for 24 and $48 \mathrm{~h}$. Cell viability was quantified through MTT assay in three independent experiments. The differences between untreated and SICAM-1 stimulated cells were considered statistically significant at ${ }^{*} p<0.05$ using Student's $t$-test. Differences between CLX-untreated versus CLX-treated cells were considered significant at ${ }^{\#} p<0.05$ and ${ }^{\#} p<0.01$ using Student's $t$-test.

A

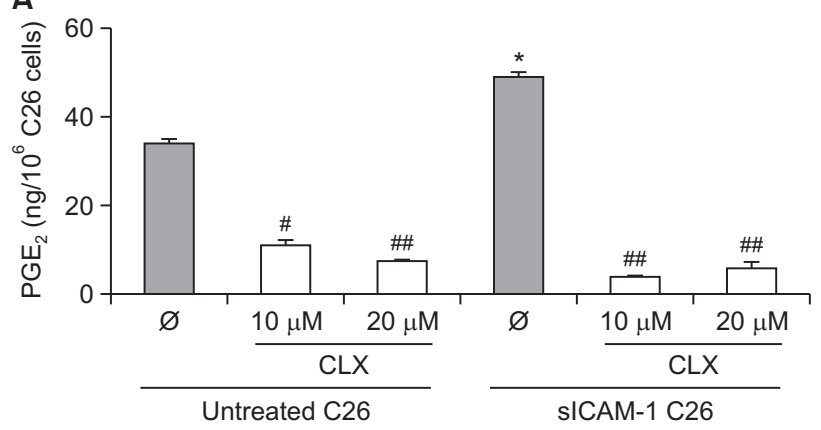

C

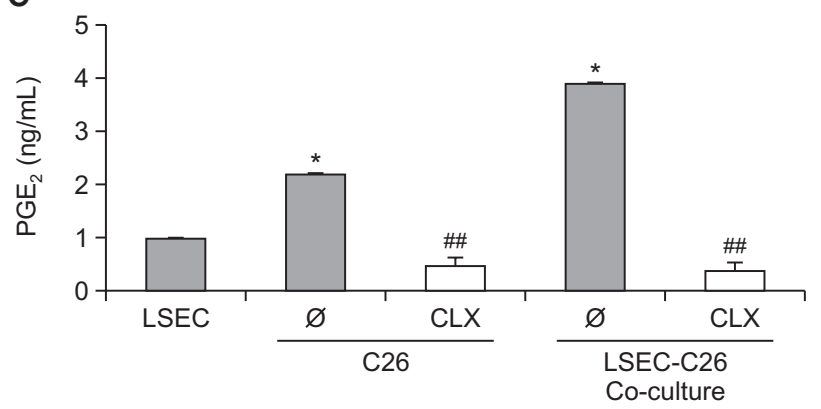

B

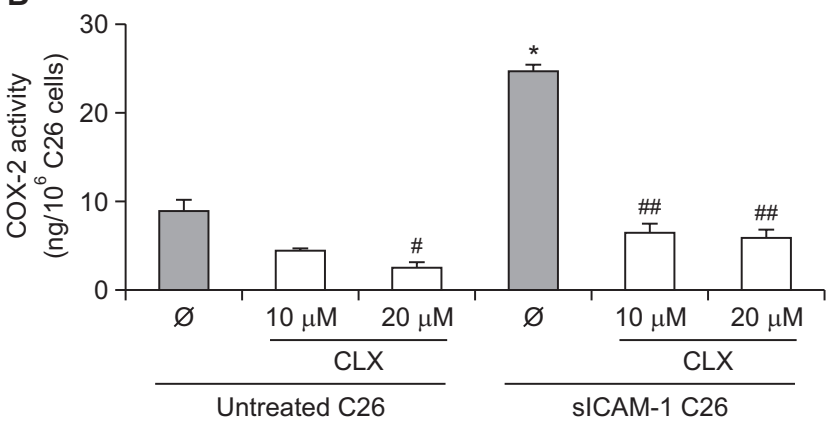

D

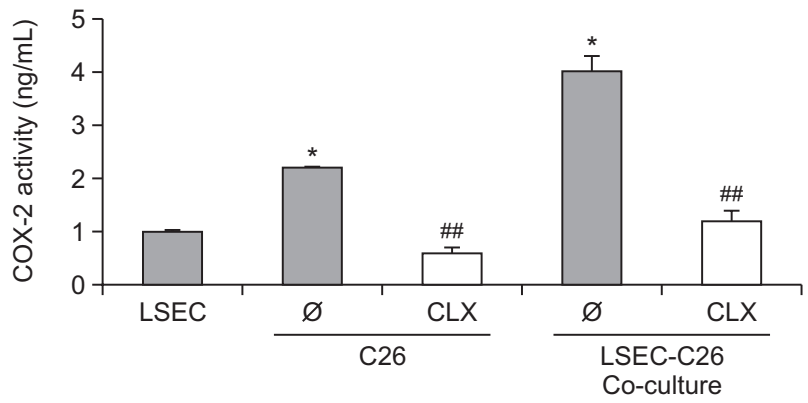

Fig. 2. Measurement of $\mathrm{PGE}_{2}$ secretion and COX-2 activity in cell cultures. (A, B) C26 monocultures were stimulated with $200 \mathrm{ng} / \mathrm{mL}$ slCAM-1 and treated with 10 and $20 \mu \mathrm{M} \mathrm{CLX}$ for the analysis of PGE 2 production and COX-2 activity. (C, D) For the coculture experiments, C26 cells were treated with CLX before culturing them with freshly isolated primary LSECs for $6 \mathrm{~h}$. Afterwards, the supernatants were collected and analyzed for $\mathrm{PGE}_{2}$ concentration and COX-2 activity using ELISA and arachidonic acid metabolization, respectively. Differences between untreated and SICAM-1-stimulated cells were considered statistically significant at ${ }^{*} p<0.05$ using Student's $t$-test. Differences between CLX-untreated versus CLX-treated cells were considered significant at ${ }^{\#} p<0.05$ and ${ }^{\#} p<0.01$ using Student's $t$-test.

\section{C26 cells increase the migratory potential of LSECs and HSCs via tumor COX-2}

The recruitment of LSECs and HSCs is a crucial event for the establishment and growth of experimental CRC tumors (Vidal-Vanaclocha, 2011). We have previously shown that C26 secretomes promote LSEC and HSC migration, and that sICAM-1 pretreatment of tumor cells enhanced this effect (Benedicto et al., 2019). Fig. 3 shows that the promigratory effect exerted by tumor cells on LSECs and HSCs is partly mediated by tumor COX-2. Thus, tumor cell pretreatment with CLX reverted the increased migration of C26-secretome-treated LSEC s and HSCs compared with that of untreated LSECs and HSCs. Hence, COX-2 arises as a regulator of the interaction between tumor cells and hepatic non-parenchymal cells. As mentioned before, this mechanism may account for the initial response of sinusoidal cells to the tumor, which is a limiting step for metastatic colonization of the liver by $\mathrm{CRC}$ in vivo.

To further confirm the involvement of COX-2 on the promigratory effect exerted by sICAM-1-activated C26 cells, we carried out in vitro wound healing assays on two non-liver cell 
A

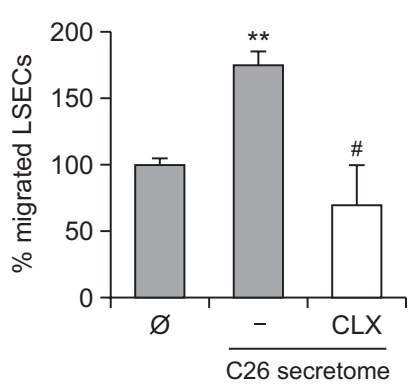

B

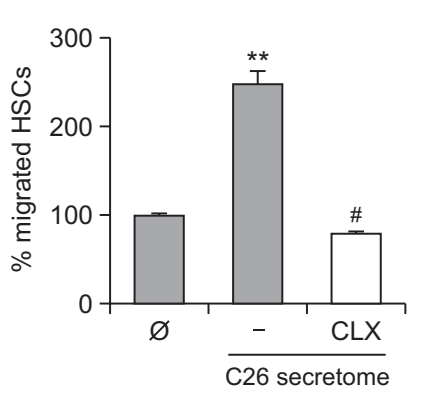

C

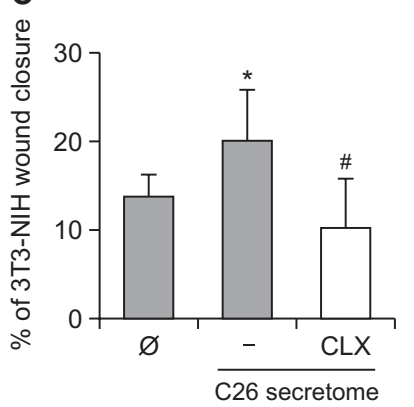

D

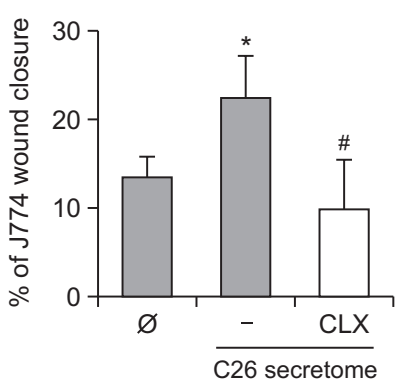

Fig. 3. The effect of COX-2 inhibition in tumor-mediated liver sinusoidal endothelial cell (LSEC) and hepatic stellate cell (HSC) migration. (A, B) Freshly isolated primary LSECs and HSCs were cultured in modified Boden chambers and were stimulated with either C26 secretomes or CLX-treated C26 secretomes for $24 \mathrm{~h}$. Afterwards, the inserts were fixed and stained with crystal violet for the quantification of migrated LSECs and HSCs. (C, D) NIH 3T3 and J774.1 were plated in 24-well plates and stimulated with C26 or CLX-treated C26 secretomes for 24 $\mathrm{h}$ after creating a scratch. Closure of this area by each cell line was quantified through ImageJ software. Differences between the control cells versus C26-secretome-stimulated cells were considered statistically significant at ${ }^{*} p<0.05$ and ${ }^{* *} p<0.01$ using Student's $t$-test. Differences between C26-secretome-activated versus C26-activated and CLX-treated cells were considered significant at $\# p<0.05$ by Student's $t$ test.

A

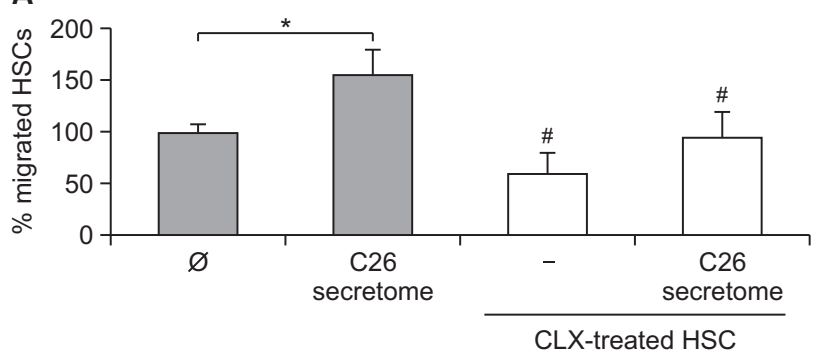

C

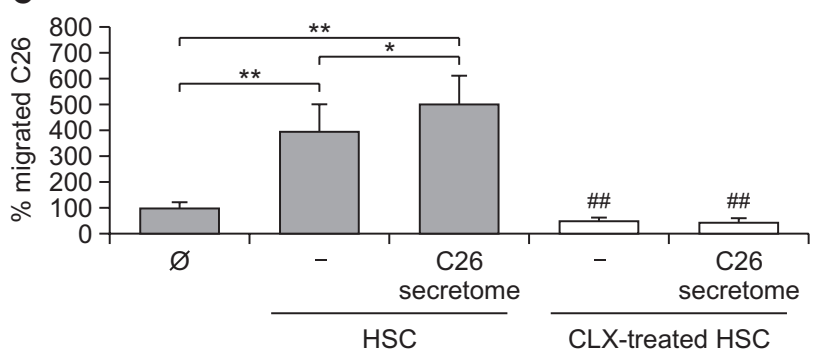

B

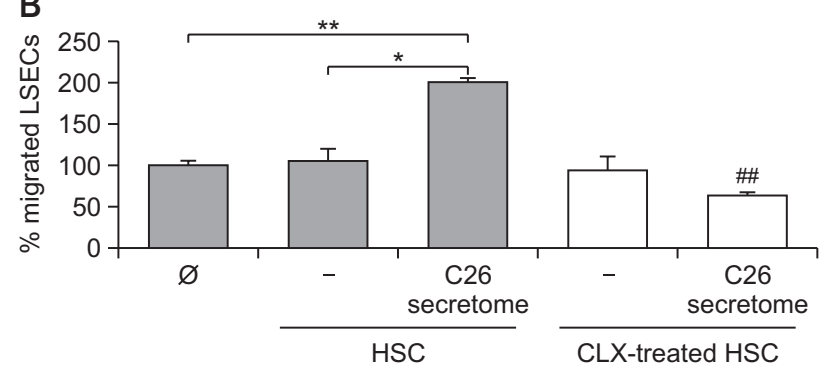

D

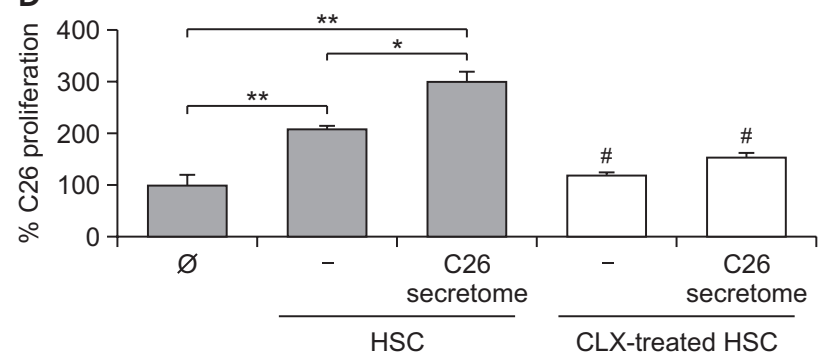

Fig. 4. Analysis of HSCs' protumoral effect upon COX-2 inhibition. COX-2 was inhibited in freshly isolated primary HSCs, and several processes linked with tumor progression were analyzed. CLX-treated HSCs were stimulated with C26 secretomes and their migration was analyzed (A). HSCs were treated with CLX and stimulated with C26 secretomes. Afterwards, soluble factors released by HSCs were collected. The migration of LSECs (B), C26 (C), and the proliferation of C26 (D) was measured in response to CLX-treated and C26-secretome-activated HSCs secretomes. Differences between untreated versus HSCs or C26-secretome-activated cells were considered statistically significant at ${ }^{*} p<0.05$ and ${ }^{* *} p<0.01$ using Student's $t$-test. Differences between HSC or C26-secretome-stimulated HSCs versus CLX-treated HSC or C26-secretome-stimulated HSC-treated cells were considered significant at ${ }^{\#} p<0.05$ and ${ }^{\# \#} p<0.01$ using Student's $t$-test.

populations, NIH 3T3 fibroblasts and J774A.1 macrophagelike cells. As observed in Fig. 3B, 3C, both cell lines responded similarly, as did primary LSECs and HSCs. Afresh, tumor cell COX-2 inhibition reduced the ability of C26 secretomes to promote the migration of both cell lines (Fig. 3C, 3D), as noted for primary LSECs and HSCs.

\section{The prometastatic response of HSC is COX-2-dependent}

Although the role of COX-2 in tumor cells has been extensively studied, the involvement of hepatic COX-2 activity dur- ing liver metastasis remains mostly unexplored. As HSCs are recruited by tumor cell secretomes to liver foci shortly after their establishment (Olaso et al., 1997), we next analyzed the effect of COX-2 inhibition on the migratory potential of HSCs. As shown in Fig. 4A, the inhibition of HSC COX-2 by CLX abrogated both basal and C26 secretome-induced HSC migration. As previously reported (Olaso et al., 2003), tumor-activated HSCs promote LSEC migration. Interestingly, COX-2 inhibition in HSCs reduced the ability of C26secretome-activated HSCs to stimulate LSEC migration, reducing LSEC migration 

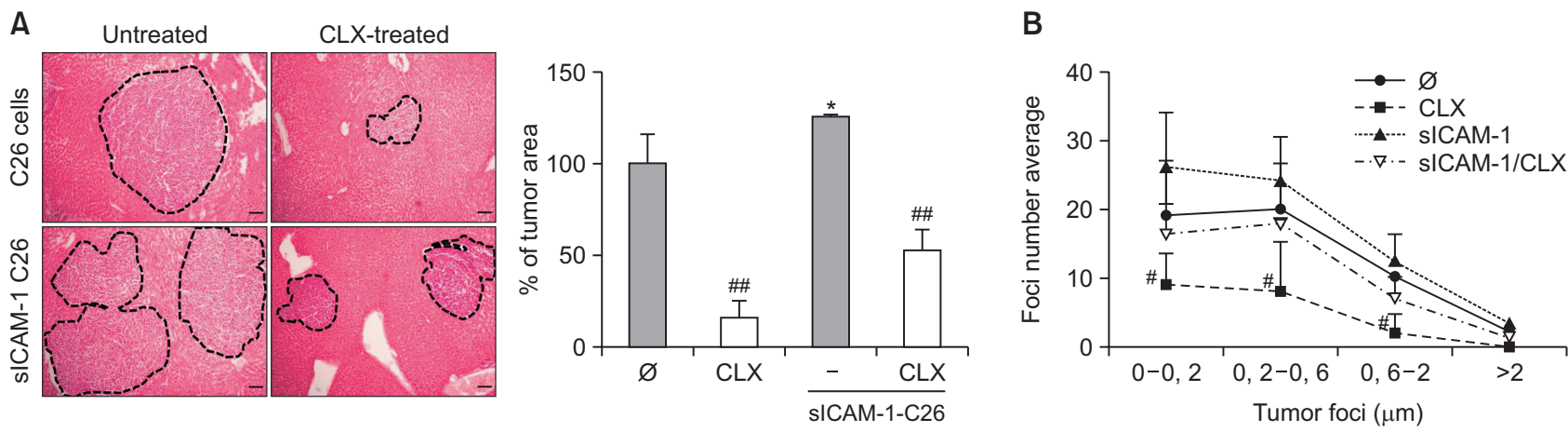

Fig. 5. Liver metastasis development in response to sICAM-1 stimulation and COX-2 blockade. C26 tumor cells were stimulated or not with sICAM-1, and injected in the distal pole of the spleen in mice. Mice were treated with oral cleavage of CLX for 14 days, and the development of liver metastasis was quantified in terms of metastatic area and foci number and size (A, B). The levels of VEGF and PGE ${ }_{2}$ were analyzed in the plasma isolated from the portal blood seven and fourteen days after tumor cell injection into the control mice, C26-injected mice and $C 26$-injected CLX-treated mice $(C, D)(n=6)$. Differences in metastatic development between untreated $C 26$ versus sICAM-1-treated C26 were considered statistically significant at ${ }^{*} p<0.05$ using Student's $t$-test. Differences between CLX-untreated versus CLX-treated cells were considered significant at ${ }^{\#} p<0.05$ and ${ }^{\# \#} p<0.01$ using Student's $t$-test.

to basal levels (Fig. 4B). Regarding the tumor response, HSCs stimulated the migration and proliferation of C26 cells by 4and 5-fold, respectively (Fig. 4C, 4D). This effect was dramatically reduced when COX-2 was inhibited in HSCs. Interestingly, pre-activation of HSCs with $\mathrm{C} 26$ secretomes increased the stimulatory ability of HSC in the migration and proliferation of tumor cells. In line with previous results, the inhibition of COX-2 in C26-secretome-activated HSCs abrogated protumoral effect of HSCs on C26 cells (Fig. 4C, 4D).

\section{ICAM-1 pretreatment of CRC cells boosts liver metastasis through COX-2}

Next, we investigated the role of sinusoidal endotheliumderived ICAM- 1 and the subsequent increase in COX-2 activity in tumor cells during experimental liver metastasis of CRC. To mimic the activation of tumor cells when interacting with LSEC ICAM-1, tumor cells were treated with SICAM-1 prior to their injection into mice.

We have previously demonstrated that in vitro sICAM-1 pretreatment increases the metastatic potential of C26 cells (Benedicto et al., 2019). Fig. 5A shows the in vivo administration of CLX to block tumor microenvironment development due to COX-2-impaired C26 cell liver metastasis compared with untreated C26 cells. Interestingly, CLX reduced the metastatic burden in the livers of mice injected with either C26 cells or sICAM-1 preactivated C26 cells (Fig. 5A), thereby eliminating the SICAM-1-mediated increased aggressiveness of tumor cells. As observed in Fig. 5B, CLX inhibited tumor growth independent of the tumor foci developmental status, ranging from avascular microfoci to larger ones that were at the vascular stage of growth. CLX also reduced the total area occupied by different size foci.

Interestingly, tumor microenvironment COX-2 inhibition reduced both the $\mathrm{PGE}_{2}$ and VEGF concentrations in the portal blood serum of tumor-bearing mice 14 days after tumor cell injection (Supplementary Fig. 2). It is worth noting that, at this late stage of tumor progression, COX-2 inhibition led to the restoration of portal $\mathrm{PGE}_{2}$ levels to those observed in healthy animals. Even though the effects on the portal VEGF concentration were less notorious, they were shown to be significant 14 days after tumor cell injection into mice treated with CLX.

\section{COX-2 blockade reduces the protumorigenic microenvironment promoted by the ICAM-1 stimulation of C26 cells}

Next, we analyzed whether CLX inhibition modified the stromal compartment of metastatic foci. In line with our previous work (Benedicto et al., 2019), the pre-stimulation of C26 cells with SICAM-1 increased the recruitment of ASMA expressing cells to metastatic foci developed in the livers by $50 \%$, the majority of them being activated HSCs (Raza et al., 2010), compared with those observed in the livers of mice injected with untreated cells. In line with the in vitro observations, the COX-2 blockade abrogated this effect, restoring the numbers of ASMA-positive cells recruited to metastatic foci back to those numbers observed in the livers from control mice (Fig. $6 \mathrm{~A}$ ). A similar phenomenon was reported regarding tumor foci infiltration by F4/80 positive macrophages (Fig. 6B). Next, we analyzed fibrillar collagen accumulation as a sign of the desmoplastic response developed during C26 tumor progression. Collagen synthesis within the tumor stroma was stimulated two-fold in the metastatic foci present in the livers collected from mice injected with sICAM-1-activated C26 cells. Interestingly, COX-2 inhibition reversed the synthesis of fibrillar collagen to the levels observed in the metastatic foci of basal C26injected mice (Fig. 6C). The reduction in the tumor stromal compartment and the stromal-mediated extracellular matrix (ECM) reorganization within the metastatic foci was correlated with tumor foci size.

\section{DISCUSSION}

COX-2 has been linked with CRC and with the metastatic spread of cancer cells to the liver (Yu et al., 2005). However, little is known about the regulation of this enzyme during the early steps of liver colonization and how it influences the liver prometastatic response. In this study, we uncovered the relevance of tumor COX-2 as a catalyst of the protumoral cascade mediated by ICAM-1 during tumor cell/LSEC crosstalk. To demonstrate this, we implemented in vitro approaches using freshly isolated LSECs and HSCs in order to achieve the most reliable in vitro approach. We conducted in vitro coculture ex- 
A
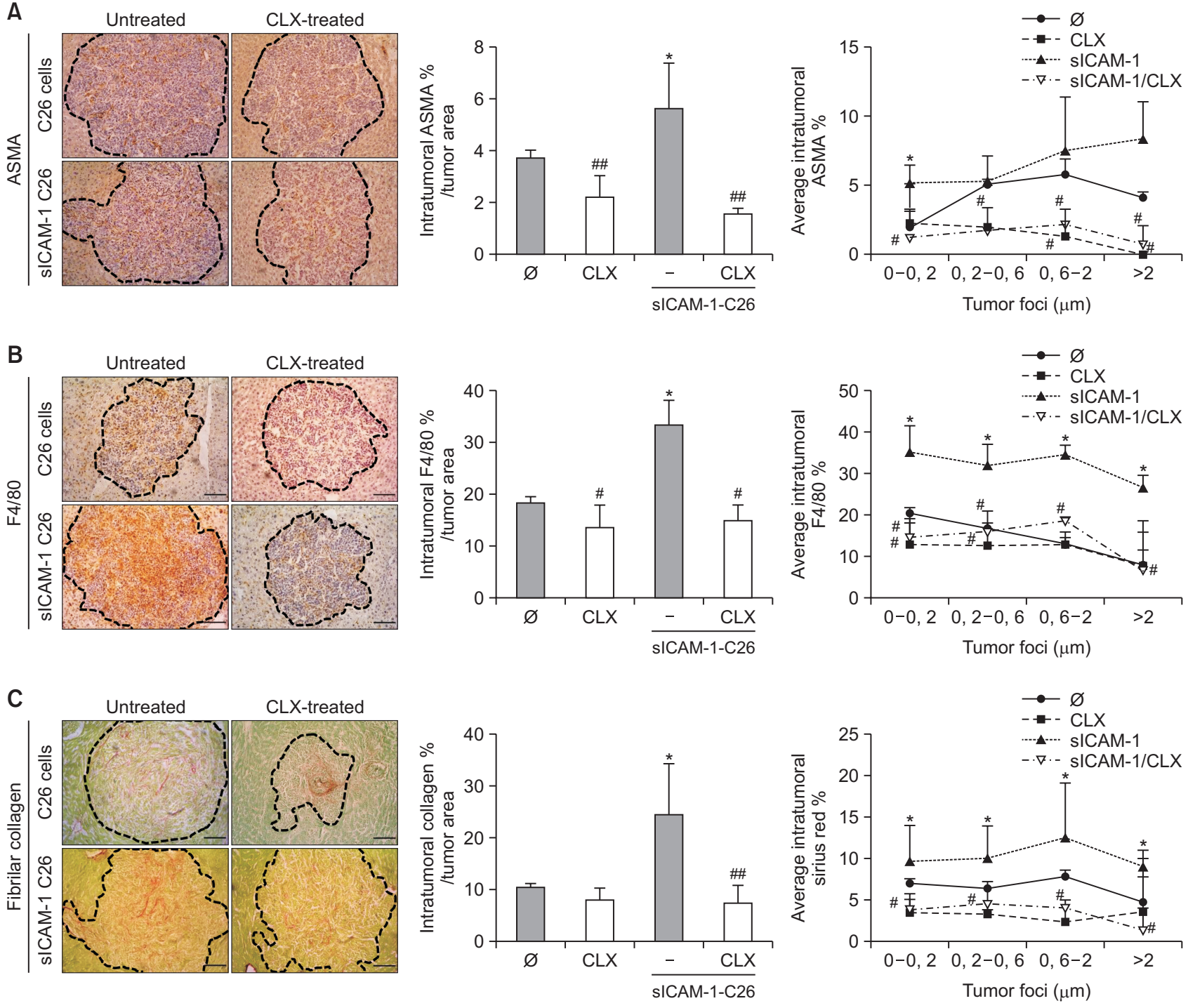

Fig. 6. Differences in intratumor stromal compartment of liver metastasis in sICAM-1- and CLX-treated mice. The infiltration of ASMA- and F4/80-expressing cells was quantified in the metastatic foci of mice under different treatment routines (A, B). Furthermore, the accumulation of intratumoral fibrillar collagen was analyzed $(C)(n=6)$. Differences between the untreated C26 versus sICAM-1-treated C26 cell intratumoral expression of ASMA, F4/80, and collagen were considered statistically significant for ${ }^{*} p<0.05$. Differences between CLX-untreated versus CLX-treated animals were considered significant at ${ }^{\#} p<0.05$ and ${ }^{\# \#} p<0.01$ using Student's $t$-test.

periments of tumor cells and LSECs; migration assays of tumor cells, LSECs, and HSCs under different conditions; and inhibited COX-2 in both cancer cells and HSCs using CLX. We confirmed the in vitro results using the in vivo orthotopic liver metastasis model of CRC. Cancer cells were activated with sICAM-1, and mice were treated with CLX to inhibit COX-2 during metastatic development. We further analyzed the metastatic stromal compartment within the tumor foci, quantifying the recruitment of ASMA expressing activated fibroblasts, F4/80-positive macrophages, and the accumulation of fibrillar collagen. Our results support the idea that after the interaction of cancer cells with LSECs via ICAM-1, a prometastatic response is triggered in the liver, mediated not only by tumor COX-2 but also by COX-2 activity in HSCs (Fig. 7).

We have previously shown that cancer cell interaction with LSEC ICAM-1 in the sinusoids promotes liver metastasis. Here, we show that ICAM-1 seems to stimulate COX-2 in tumor cells, promoting cancer cell proliferation. Interestingly, COX-2 is the main mediator of $\mathrm{PGE}_{2}$, which is elevated in the blood plasma of cancer patients suffering from CRC liver metastasis (Narisawa et al., 1990). In line with this report, our studies show that the first interaction between metastatic cancer cells and LSECs gives rise to increased COX-2 activity and subsequent $\mathrm{PGE}_{2}$ in an in vitro setting. This result was further confirmed by the SICAM-1 stimulation of cancer cells.

It is well known that during metastatic growth, cancer cells recruit stromal cells to create a favorable microenvironment for tumor progression (Brodt, 2016). In the liver, this is represented by the recruitment of HSCs and LSECs into the nascent foci. Here, we show that COX-2 activation in cancer 


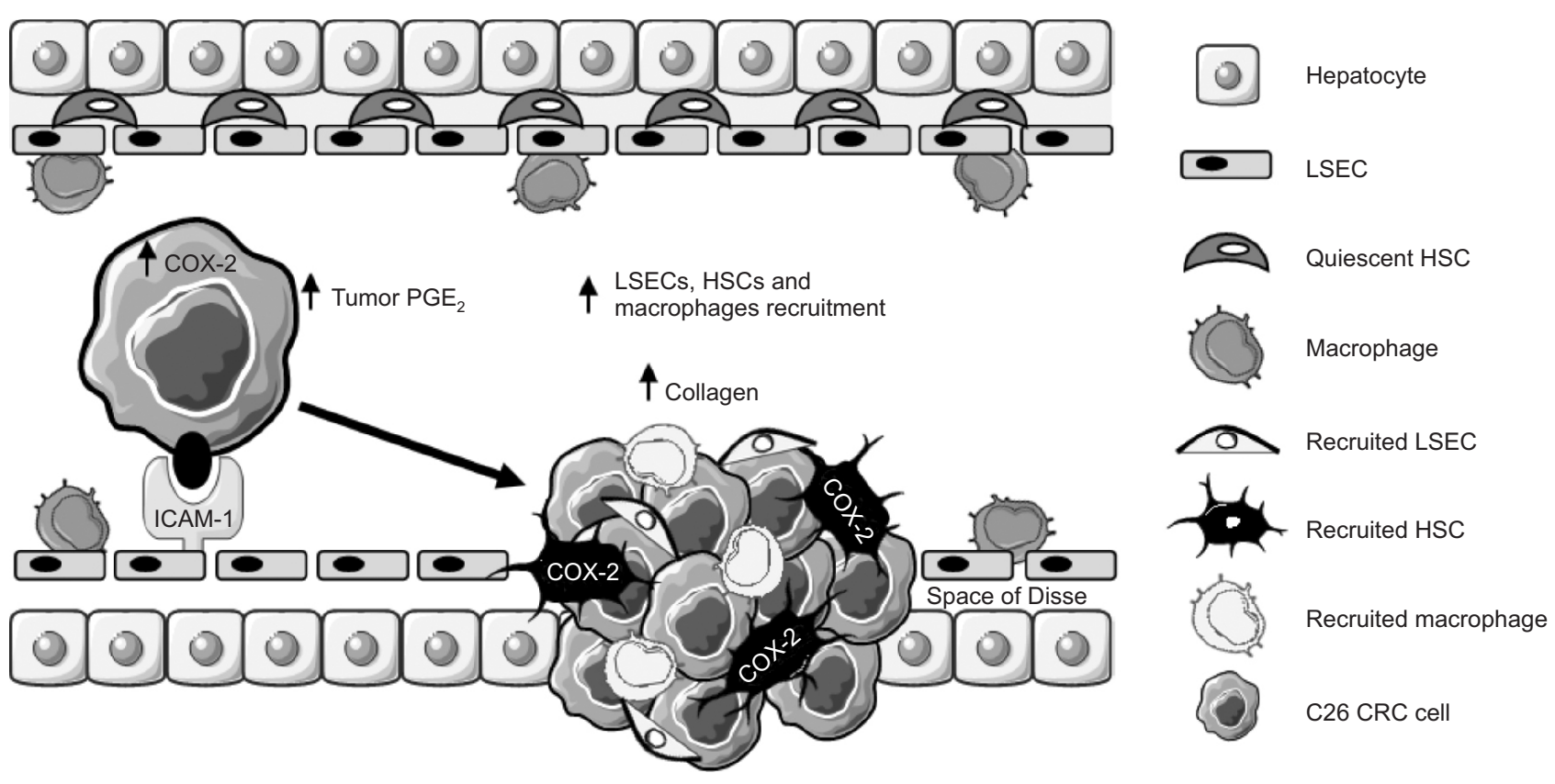

Fig. 7. Tumor COX-2 is upregulated during early stage of liver colonization. Upon tumor cell/LSEC interplay, ICAM-1 stimulates COX-2 increase and $\mathrm{PGE}_{2}$ secretion by CRC cells. COX-2 mediates the secretion of promigratory factors for LSECs, HSCs, and macrophages favoring the creation of a protumoral tumor microenvironment. Moreover, HSC COX-2 supports tumor growth, promoting LSEC migration, tumor cell proliferation, and migration. Therefore, the ICAM-1-mediated increase in COX-2 arises as a promoter of liver metastasis in CRC.

cells promotes the secretion of attractant soluble factors that induce the migration of LSECs and HSCs. Interestingly, it has been reported that endothelial cell migration and tube formation is stimulated by $\mathrm{PGE}_{2}$. This finding may explain the reported reduction in LSEC migration when these cells are stimulated with CLX-treated C26 secretomes (Jana et al., 2016; Khan et al., 2019). Moreover, COX-2 mediates the secretion of VEGF (Supplementary Fig. 1; Cianchi et al., 2005; Toomey et al., 2009), a potent promigratory factor for both LSECs and HSCs. Thus, COX-2 inhibition may account for the reduced migration of HSCs and LSECs in vitro and for recruitment to the metastatic foci in vivo. Among the recruited liver-resident cells, HSCs are involved in angiogenesis by secreting VEGF under hypoxic conditions, driving LSEC recruitment and activation (Olaso et al., 2003). Furthermore, HSCs are the primary source of fibrillar collagen in the liver, which makes them a good target for anti-fibrotic therapies (Puche et al., 2013). Moreover, HSCs express COX-2, which is upregulated during hypoxic conditions. Here, we show that COX-2 inhibition reduced the migration of C26-secretome-activated HSCs. COX2 inhibition by CLX could, in turn, reduce Akt activity on HSCs (Paik et al., 2009), which could impair HSC migration, given that Akt is involved in this process (Zhang et al., 2020).

Interestingly, COX-2 inhibition decreased the ability of C26secretome-activated HSCs to promote LSEC and C26 cell migration. This effect could be linked with the reduced VEGF secretion by CLX-treated HSCs, as VEGF mediates the migration of LSECs and CRC cells (Valcárcel et al., 2008; Bhattacharya et al., 2017; Benedicto et al., 2019). Furthermore, VEGF reduction in CLX-treated HSCs may also explain their impaired ability to promote C26 cell proliferation compared with that of C26-secretome-activated HSCs, as this mediator increases gastric cancer cell proliferation (Ma et al., 2019).
In concordance with our in vitro data, the analysis of in vivo liver metastasis by ICAM-1-activated C26 cells followed the same reduction trend under CLX treatment. In line with our previous reports, sICAM-1 pretreatment of tumor cells increased metastatic growth in the liver. Interestingly, COX-2 inhibition not only reduced liver colonization by untreated $\mathrm{C} 26$ cells but also diminished the prometastatic effect of those treated with sICAM-1. In the tumor microenvironment, a decrease in $\mathrm{PGE}_{2}$ and VEGF levels in CLX-treated mice, which may be derived from C26 cells and HSCs, among others, was correlated with a reduction in tumor development (Supplementary Fig. 2). Therefore, the recruitment of LSECs to nascent foci may be abrogated, thus impairing tumor angiogenesis. Furthermore, myeloid-derived suppressor cells (MDSC), an immune population able to impair immune response, are accumulated during liver metastasis (Burga et al., 2015; Benedicto et al., 2018), and favor CRC liver metastasis and angiogenesis (Lin et al., 2019; Vetsika et al., 2019). It has been documented that both VEGF and $\mathrm{PGE}_{2}$ drive the accumulation and differentiation of MDSCs in the tumor microenvironment (Obermajer et al., 2011; Horikawa et al., 2017). Both $\mathrm{PGE}_{2}$ and VEGF levels are reduced in CLX-treated mice, which, in turn, may prevent MDSC recruitment and differentiation, as reported in several cancer models upon COX-2 inhibition (Veltman et al., 2010; Fujita et al., 2011; Xu et al., 2014). Moreover, activated HSCs drive the accumulation of MDSC during liver cancer through COX-2 (Xu et al., 2016). We hypothesize that CLX treatment may block this recruitment into the liver of this protumoral cell population, slowing down tumor growth.

Regarding stromal cell interaction, HSCs and macrophages are key players that drive several steps in the development of liver metastasis (Olaso et al., 2003; Keirsse et al., 2018). Thus, their recruitment seems to be critical for disease progres- 
sion. COX-2 inhibition significantly reduced the recruitment of HSCs and macrophages into metastatic foci. Interestingly, COX-2 blockade inhibited ICAM-1-mediated augmentation of the stromal reaction. We hypothesize that reduced protumoral stimulus generated by both tumor cells and tumor-activated stromal cells impairs the recruitment of HSCs and macrophages. In fact, the observed reduction in VEGF levels in the blood plasma may explain the reported reduction in ASMAexpressing cell recruitment into the metastatic foci as VEGF mediates both the migration and proliferation of HSCs (Olaso et al., 2003; Liu et al., 2009). Regarding the implication of macrophages in the liver's reaction to tumor cell colonization and growth, the decreased inflammatory response generated by reduced tumor growth may account for the impaired F4/80positive cell infiltration in metastatic foci. Furthermore, activated HSCs constitute a significant source of CXCL12, which mediates the recruitment of CXCR4-expressing MDSCs (Xu et al., 2019) into the liver. In line with this process, cancerassociated fibroblasts (CAFs) drive the accumulation and differentiation of monocytes into M2 macrophages, which may also occur in the liver (Li et al., 2019).

The obtained data support the role of tumor COX-2 as a mediator in the early steps of liver metastasis, which both increases the response of tumor cells to ICAM-1 and drives the prometastatic phenotype of HSCs. COX-2 amplifies the creation of a favorable niche in the liver, leading to metastatic growth. These results uncover novel roles for COX-2 while suggesting a combination approach with chemotherapy drugs to reduce metastatic lesions of $\mathrm{CRC}$.

\section{CONFLICT OF INTEREST}

The authors declare no conflicts of interest.

\section{ACKNOWLEDGMENTS}

This work was supported by the Department of Industry and Research of the Basque Government SAIOTEK SPE12UN075 and S-PE11UN043 to B.A., IT-487-09 to E.O., and by the Spanish Science and Technology Ministry MINECOR18/P32.

\section{REFERENCES}

Arteta, B., Lasuen, N., Lopategi, A., Sveinbjörnsson, B., Smedsrød, B. and Vidal-Vanaclocha, F. (2010) Colon carcinoma cell interaction with liver sinusoidal endothelium inhibits organ-specific antitumor immunity through interleukin-1-induced mannose receptor in mice. Hepatology 51, 2172-2182.

Benedicto, A., Marquez, J., Herrero, A., Olaso, E., Kolaczkowska, E. and Arteta, B. (2017) Decreased expression of the $\beta 2$ integrin on tumor cells is associated with a reduction in liver metastasis of colorectal cancer in mice. BMC Cancer 17, 827.

Benedicto, A., Romayor, I. and Arteta, B. (2018) CXCR4 receptor blockage reduces the contribution of tumor and stromal cells to the metastatic growth in the liver. Oncol. Rep. 39, 2022-2030.

Benedicto, A., Herrero, A., Romayor, I., Marquez, J., Smedsrød, B., Olaso, E. and Arteta, B. (2019) Liver sinusoidal endothelial cell ICAM-1 mediated tumor/endothelial crosstalk drives the development of liver metastasis by initiating inflammatory and angiogenic responses. Sci. Rep. 9, 13111.
Bhattacharya, R., Fan, F., Wang, R., Ye, X., Xia, L., Boulbes, D. and Ellis, L. M. (2017) Intracrine VEGF signalling mediates colorectal cancer cell migration and invasion. Br. J. Cancer 117, 848-855.

Brodt, P. (2016) Role of the microenvironment in liver metastasis: from pre- to prometastatic niches. Clin. Cancer Res. 22, 5971-5982.

Burga, R. A., Thorn, M., Point, G. R., Guha, P., Nguyen, C. T., Licata, L. A., DeMatteo, R. P., Ayala, A., Joseph Espat, N., Junghans, R. P. and Katz, S. C. (2015) Liver myeloid-derived suppressor cells expand in response to liver metastases in mice and inhibit the antitumor efficacy of anti-CEA CAR-T. Cancer Immunol. Immunother. 64, 817-829.

Chen, W. S., Wei, S. J., Liu, J. M., Hsiao, M., Kou-Lin, J. and Yang, W. K. (2001) Tumor invasiveness and liver metastasis of colon cancer cells correlated with cyclooxygenase-2 (COX-2) expression and inhibited by a COX-2-selective inhibitor, etodolac. Int. J. Cancer $\mathbf{9 1}$, 894-899.

Cianchi, F., Cortesini, C., Schiavone, N., Perna, F., Magnelli, L., Fanti, E., Bani, D., Messerini, L., Fabbroni, V., Perigli, G., Capaccioli, S. and Masini, E. (2005) The role of cyclooxygenase-2 in mediating the effects of histamine on cell proliferation and vascular endothelial growth factor production in colorectal cancer. Clin. Cancer Res. 11, 6807-6815.

Fujita, M., Kohanbash, G., Fellows-Mayle, W., Hamilton, R. L., Komohara, Y., Decker, S. A., Ohlfest, J. R. and Okada, H. (2011) COX-2 blockade suppresses gliomagenesis by inhibiting myeloid-derived suppressor cells. Cancer Res. 71, 2664-2674.

Greenhough, A., Smartt, H. J. M., Moore, A. E., Roberts, H. R., Williams, A. C., Paraskeva, C. and Kaidi, A. (2009) The COX-2/PGE2 pathway: key roles in the hallmarks of cancer and adaptation to the tumour microenvironment. Carcinogenesis 30, 377-386.

Horikawa, N., Abiko, K., Matsumura, N., Hamanishi, J., Baba, T., Yamaguchi, K., Yoshioka, Y., Koshiyama, M. and Konishi, I. (2017) Expression of vascular endothelial growth factor in ovarian cancer inhibits tumor immunity through the accumulation of myeloid-derived suppressor cells. Clin. Cancer Res. 23, 587-599.

Jana, S., Chatterjee, K., Ray, A. K., DasMahapatra, P. and Swarnakar, S. (2016) Regulation of matrix metalloproteinase-2 activity by COX-2-PGE2-pAKT axis promotes angiogenesis in endometriosis. PLOS ONE 11, e0163540.

Keirsse, J., Van Damme, H., Geeraerts, X., Beschin, A., Raes, G. and Van Ginderachter, J. A. (2018) The role of hepatic macrophages in liver metastasis. Cell. Immunol. 330, 202-215.

Khan, G. A., Bhagat, S. and Alam, M. I. (2019) PGE 2 -induced migration of human brain endothelial cell is mediated though protein kinase A in cooperation of EP receptors. J. Leukoc. Biol. 105, 705717.

Khatib, A. M., Fallavollita, L., Wancewicz, E. V., Monia, B. P. and Brodt, P. (2002) Inhibition of hepatic endothelial E-selectin expression by C-raf antisense oligonucleotides blocks colorectal carcinoma liver metastasis. Cancer Res. 62, 5393-5398.

Li, X., Bu, W., Meng, L., Liu, X., Wang, S., Jiang, L., Ren, M., Fan, Y. and Sun, H. (2019) CXCL12/CXCR4 pathway orchestrates CSClike properties by CAF recruited tumor associated macrophage in OSCC. Exp. Cell Res. 378, 131-138.

Lin, Q., Ren, L., Jian, M., Xu, P., Li, J., Zheng, P., Feng, Q., Yang, L., Ji, M., Wei, Y. and Xu, J. (2019) The mechanism of the premetastatic niche facilitating colorectal cancer liver metastasis generated from myeloid-derived suppressor cells induced by the S1PR1-STAT3 signaling pathway. Cell Death Dis. 10, 693.

Liu, Y., Lui, E. L. H., Friedman, S. L., Li, L., Ye, T., Chen, Y., Poon, R. T., Wo, J., Kok, T. W. and Fan, S. T. (2009) PTK787/ZK22258 attenuates stellate cell activation and hepatic fibrosis in vivo by inhibiting VEGF signaling. Lab. Investig. 89, 209-221.

Ma, F., Zhang, B., Ji, S., Hu, H., Kong, Y., Hua, Y. and Luo, S. (2019) Hypoxic macrophage-derived VEGF promotes proliferation and invasion of gastric cancer cells. Dig. Dis. Sci. 64, 3154-3163.

Nagatsuka, I., Yamada, N., Shimizu, S., Ohira, M., Nishino, H., Seki, S. and Hirakawa, K. (2002) Inhibitory effect of a selective cyclooxygenase-2 inhibitor on liver metastasis of colon cancer. Int. J. Cancer 100, 515-519.

Narisawa, T., Kusaka, H., Yamazaki, Y., Takahashi, M., Koyama, H., Koyama, K., Fukaura, Y. and Wakizaka, A. (1990) Relationship be- 
tween blood plasma prostaglandin E2 and liver and lung metastases in colorectal cancer. Dis. Colon Rectum 33, 840-845.

Obermajer, N., Muthuswamy, R., Lesnock, J., Edwards, R.P. and Kalinski, P. (2011) Positive feedback between PGE2 and COX2 redirects the differentiation of human dendritic cells toward stable myeloid-derived suppressor cells. Blood 118, 5498-5505.

Olaso, E., Santisteban, A., Bidaurrazaga, J., Gressner, A. M., Rosenbaum, J. and Vidal-Vanaclocha, F. (1997) Tumor-dependent activation of rodent hepatic stellate cells during experimental melanoma metastasis. Hepatology 26, 634-642.

Olaso, E., Salado, C., Egilegor, E., Gutierrez, V., Santisteban, A., Sancho-Bru, P., Friedman, S. L. and Vidal-Vanaclocha, F. (2003) Proangiogenic role of tumor-activated hepatic stellate cells in experimental melanoma metastasis. Hepatology 37, 674-685.

Paik, Y. H., Kim, J. K., Lee, J. I., Kang, S. H., Kim, D. Y., An, S. H., Lee, S. J., Lee, D. K., Han, K. H., Chon, C. Y., Lee, S. I., Lee, K. S. and Brenner, D. A. (2009) Celecoxib induces hepatic stellate cell apoptosis through inhibition of Akt activation and suppresses hepatic fibrosis in rats. Gut 58, 1517-1527.

Puche, J. E., Lee, Y. A., Jiao, J., Aloman, C., Fiel, M. I., Muñoz, U., Kraus, T., Lee, T., Yee, H. F. and Friedman, S. L. (2013) A novel murine model to deplete hepatic stellate cells uncovers their role in amplifying liver damage in mice. Hepatology 57, 339-350.

Raza, A., Franklin, M. J. and Dudek, A. Z. (2010) Pericytes and vessel maturation during tumor angiogenesis and metastasis. Am. J. Hematol. 85, 593-598.

Shoji, T., Konno, H., Tanaka, T., Sakaguchi, T., Sunayama, K., Baba, M., Kamiya, K., Ohta, M., Kaneko, T., Igarashi, A. and Nakamura, S. (2003) Orthotopic implantation of a colon cancer xenograft induces high expression of cyclooxygenase-2. Cancer Lett. 195, 235-241.

Siegel, R. L., Miller, K. D. and Jemal, A. (2019) Cancer statistics, 2019. CA. Cancer J. Clin. 69, 7-34

Smedsrod, B. and Pertoft, H. (1985) Preparation of pure hepatocytes and reticuloendothelial cells in high yield from a single rat liver by means of Percoll centrifugation and selective adherence. J. Leukoc. Biol. 38, 213-230.

Toomey, D. P., Murphy, J. F. and Conlon, K. C. (2009) COX-2, vegf and tumour angiogenesis. Surgeon 7, 174-180.

Valcárcel, M., Arteta, B., Jaureguibeitia, A., Lopategi, A., Martínez, I., Mendoza, L., Muruzabal, F. J., Salado, C. and Vidal-Vanaclocha, F. (2008) Three-dimensional growth as multicellular spheroid activates the proangiogenic phenotype of colorectal carcinoma cells via LFA-1-dependent VEGF: implications on hepatic micrometastasis. J. Transl. Med. 6, 57. van der Pool, A. E. M., Damhuis, R. A., ljzermans, J. N. M., de Wilt, J. H. W., Eggermont, A. M. M., Kranse, R. and Verhoef, C. (2012) Trends in incidence, treatment and survival of patients with stage IV colorectal cancer: a population-based series. Colorectal Dis. 14, 56-61.

Veltman, J. D., Lambers, M. E., van Nimwegen, M., Hendriks, R. W., Hoogsteden, H. C., Aerts, J. G. and Hegmans, J. P. (2010) COX2 inhibition improves immunotherapy and is associated with decreased numbers of myeloid-derived suppressor cells in mesothelioma. Celecoxib influences MDSC function. BMC Cancer 10, 464.

Vetsika, E. K., Koukos, A. and Kotsakis, A. (2019) Myeloid-derived suppressor cells: major figures that shape the immunosuppressive and angiogenic network in cancer. Cells 8, 1647.

Vidal-Vanaclocha, F. (2011) The liver prometastatic reaction of cancer patients: Implications for microenvironment-dependent colon cancer gene regulation. Cancer Microenviron. 4, 163-180.

Volpes, R., van den Oord, J. J. and Desmet, V. J. (1990) Immunohistochemical study of adhesion molecules in liver inflammation. Hepatology 12, 59-65.

Xu, L., Stevens, J., Hilton, M. B., Seaman, S., Conrads, T. P., Veenstra, T. D., Logsdon, D., Morris, H., Swing, D. A., Patel, N. L., Kalen, J., Haines, D. C., Zudaire, E. and St Croix, B. (2014) COX-2 inhibition potentiates antiangiogenic cancer therapy and prevents metastasis in preclinical models. Sci. Transl. Med. 6, 242 ra84.

Xu, Y., Zhao, W., Xu, J., Li, J., Hong, Z., Yin, Z. and Wang, X. (2016) Activated hepatic stellate cells promote liver cancer by induction of myeloid-derived suppressor cells through cyclooxygenase-2. Oncotarget 7, 8866-8878.

Xu, Y., Fang, F., Jiao, H., Zheng, X., Huang, L., Yi, X. and Zhao, W. (2019) Activated hepatic stellate cells regulate MDSC migration through the SDF-1/CXCR4 axis in an orthotopic mouse model of hepatocellular carcinoma. Cancer Immunol. Immunother. 68, 1959-1969.

Yao, M., Lam, E. C., Kelly, C. R., Zhou, W. and Wolfe, M. M. (2004) Cyclooxygenase-2 selective inhibition with NS-398 suppresses proliferation and invasiveness and delays liver metastasis in colorectal cancer. Br. J. Cancer 90, 712-719.

Yu, J. R., Wu, Y. J., Qin, Q., Lu, K. Z., Yan, S., Liu, X. S. and Zheng, S. S. (2005) Expression of cyclooxygenase-2 in gastric cancer and its relation to liver metastasis and long-term prognosis. World J. Gastroenterol. 11, 4908-4911.

Zhang, F., Xu, M., Yin, X., Guo, H., Zhang, B., Wang, Y., Xiao, J., Zou, X., Zhang, M. and Zhuge, Y. (2020) TWEAK promotes hepatic stellate cell migration through activating EGFR/Src and PI3K/AKT pathways. Cell Biol. Int. 44, 278-285. 